\title{
Trajectory prediction for future air traffic management - complex manoeuvres and taxiing
}

\author{
W. Schuster \\ w.schuster@imperial.ac.uk \\ Centre for Transport Studies \\ Imperial College \\ London \\ UK
}

\section{ABSTRACT}

The future air traffic management (ATM) concept envisaged by the Single European Sky ATM Research - SESAR - and the USA equivalent NextGen, mark a paradigm shift from the current reactive approach of ATM towards holistic strategic collaborative decision making. The core of the future ATM concept relies on common situational awareness over potentially large time-horizons, based upon the user operational intent. This is beyond human capabilities and requires the support of automation tools to predict aircraft state throughout the operation and provide support to optimal decision making long before any potential conflict may arise. This is achieved with trajectory predictors and conflict detectors and resolvers respectively. Numerous tools have been developed, typically geared towards addressing specific airborne applications. However, a comprehensive literature search suggests that none of the tools was designed to predict trajectories throughout the entire operation of an aircraft, i.e. gate-to-gate. Yet, such functionality is relevant in the holistic optimisation of aircraft operations. To address this gap, this paper builds on an existing en route trajectory prediction (TP) model and develops novel techniques to predict aircraft trajectories for the transitions between the ground- and enroute-phases of operation and for the ground-phase, thereby enabling gate-to-gate (or enroute-to-enroute) TP. The model is developed on the basis of Newtonian physics and operational procedures. Real recorded data obtained from a flight data 
record (FDR) were used to estimate some of the input parameters required by the model. The remaining parameters were taken from the BADA 3.7 model. Performance results using these flight data demonstrate that the proposed TP model has the potential to accurately predict gate-togate trajectories and to support future ATM applications such as gate-to-gate synchronisation.

\section{NOMENCLATURE}

$3 \mathrm{D}$

4D

ADS

AE

AI

ASM

ATC

ATE

ATM

BADA

CD\&R

CRT

CTE

EDM

EUROCONTROL

FAA

FCR

FO

FDR

FMS

FPA

HE

HIPER

HM

LAT

NextGen

$\mathrm{OI}$

RIGS

ROCD

RT

RTO

SESAR

TAS

TBO

TCP

TM

TMA

TOE

TP

WP three dimensional

four dimensional

aircraft dynamics system

altitude error

aircraft intent

airport surface movement

air traffic control

along track error

air traffic management

base of aircraft data (EUROCONTROL)

conflict detection and resolution

constant radius turn

cross track error

expedited descent mode

European Organisation for the Safety of Air Navigation

Federal Aviation Administration (USA)

fuel consumption rate

flight object

flight data record

flight management system

flight path angle

heading error

high performance

hold mode

look ahead time

Next Generation ATM (USA)

operational intent

required initial ground speed

rate of climb descent

regular turn

required time of overfly

Single European Sky ATM Research (EU)

true air speed

trajectory based operations

trajectory change point

turn mode

terminal manoeuvring area

time of overfly error

trajectory prediction

way point 


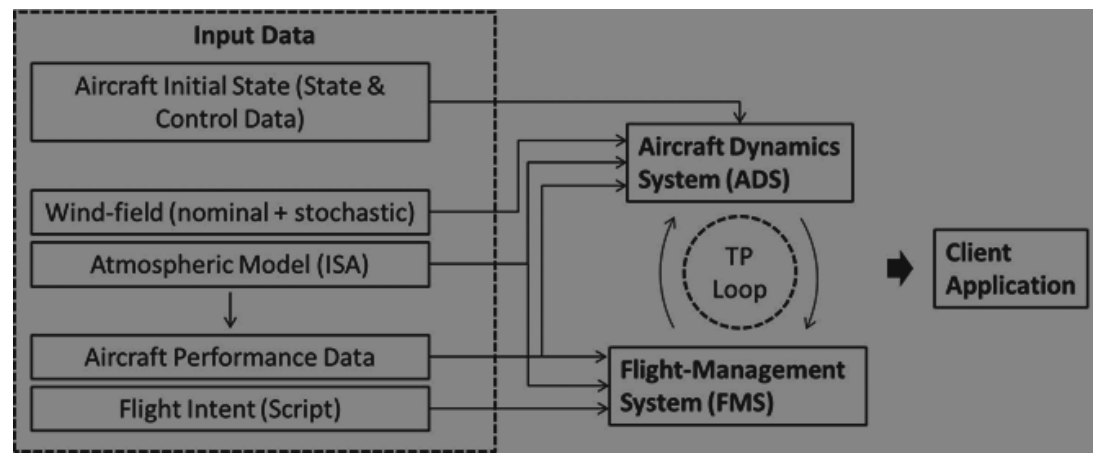

Figure 1. Flow diagram of the trajectory prediction model (adapted from Ref. 14).

\subsection{INTRODUCTION}

Current ATM is inefficient when considered from a holistic perspective. ATM is fragmented at operational, functional and technological levels and integration is required to improve efficiency (and thereby capacity) and safety, and reduce environmental impacts. This requires a more strategic approach to ATM, including better use of current technologies and the development of new ones. The Single-European-Sky ATM Research ${ }^{(1)}$ and Next Generation Air Transportation System ${ }^{(2)}$ initiatives recognise that guaranteed common situational awareness between relevant stakeholders for the entire duration of aircraft operation is key to a more efficient integration of the ATM system elements. This requires the ability to visualise the evolution of air traffic as a function of time. Gate-to-gate high-performance TP has the potential to increase capacity and efficiency, by improving the capability of air traffic control (ATC) to perform holistic strategic synchronisation and separation, rather than the current inefficient tactical process.

Conceptually, a TP tool can be divided into the following elements: the aircraft dynamics system (ADS), an input data module and a flight-management system (FMS) (see Fig. 1). The design of a TP tool is determined by its operational application. Applications such as tactical conflict detection and resolution (CD\&R) require short- (on the order of up to a few minutes look-ahead time - LAT) to medium-duration (approximately 20 minutes LAT) TP, while applications such as strategic traffic-flow optimisation require long-duration TP (with LATs on the order of hours). Numerous models have been developed, summarised in detail in Ref. 3. The mathematical models underlying each of the TP tools can be divided into four highlevel categories: point-mass models, kinematic models, kinetic models and those not falling into these three main categories. The most developed of these are the point-mass models, which predict trajectories on the basis of increasingly sophisticated and realistic models of the physics of flight. Most of these tools, including those described in Refs 4-10 were developed for short- or medium-term applications. These are not suitable for holistic strategic approaches to ATM. Other models were developed for longer LATs, including in Refs 11-13. These have the potential to support strategic ATM. However, neither is addressing trajectory prediction from a gate-to-gate perspective. Moreover, they do not model complex manoeuvres or airport surface movement. 
Ref. 14 developed a TP tool that makes increased use of aircraft intent information and improved the formulation of the mathematical model for the en route phase of flight. This paper builds on the framework of that TP tool and extends its prediction capabilities from the en route portion of operations, to a gate-to-gate prediction capability by developing a TP model for complex manoeuvres, typical of the terminal manoeuvring area (TMA), and for taxiing. The gate-to-gate TP model can be used both onboard aircraft as well as by ground-based systems. It enables the strategic as well as tactical detection and resolution of conflicts for both ground and airborne traffic over the entire duration of the operation. These features facilitate a holistic optimisation in air traffic efficiency and capacity, for example, by enabling better co-ordination of surface movement, arrival and departure traffic at airports, while minimising environmental impacts, in addition to safety assurance through short- and medium-duration conflict detection and resolution.

The paper adopts the TP structure and terminology proposed in Ref. 15, with minor modifications: flight intent will be referred to as operational intent to more adequately describe the combined ground and airborne operations. The paper describes the ADS used for the airborne phase of operation (Section 2.1) developed in Ref. 14 and develops a simple ADS model for taxiing (Section 2.2). Inputs to the ADS include the flight intent script, encapsulated in the flight object, as described in Section 3. In this respect, the paper shows that the current turn information provided in the flight plan $^{(16)}$ can be simplified and still achieve a very high accuracy. The FMS of the proposed TP model is described in Section 4, with focus on the novelties developed in this paper for complex manoeuvres (Sections 4.2.1, 4.3.1, 4.4.1 and 4.5.1) and for taxiing (Sections 4.1.2, 4.2.2, 4.3.2, 4.4.2 and 4.5.2). A key emphasis in the development of the HIgh PERformance TP (HIPER-TP) model is on accurately simulating not only the aircraft four-dimensional (4D) trajectory but also key aircraft control parameters (e.g. thrust). This is very important as different combinations of control parameters, and performance parameters, from the EUROCONTROL BADA model, Version 3.7 $7^{(17)}$, could generate the same trajectory. HIPER-TP therefore not only focuses on accuracy performance of the $4 \mathrm{D}$ position under ideal conditions, but also the accuracy performance of other key parameters, thereby making the model more operationally realistic. The performance is characterised with limited real operational data in Section 5.

\subsection{AIRCRAFT DYNAMICS SYSTEM}

\subsection{Airborne phase}

In this paper, aircraft motion for the airborne phase is modelled using a point-mass model (PMM) described in Ref. 14 and reproduced below (see Equation (1) and Fig. 2), derived from basic aerodynamics and Newtonian laws. By making the simplifying assumptions that commercial aircraft only execute benign manoeuvres and that forces in the vertical plane are in equilibrium, aircraft motion can be described by a non-linear control system with six state-variables: the threedimensional (3D) position $(x, y, h)$, the aircraft true airspeed VTAS, the aircraft heading angle $\Psi$, defined with respect to the east-direction positive counter-clockwise, and the aircraft mass $\mathrm{m}$. Aircraft dynamics is controlled by the following four control variables: engine thrust $T$, bank angle $\varphi$, flight-path angle (FPA) $\gamma$ and drag coefficient $C_{D}$ (e.g. by adjusting speed brakes). These emulate practical controls available to pilots. Additionally, aircraft dynamics is perturbed by the surrounding potentially (time) varying wind-field (i.e. it accounts for windshear), represented by a $3 \mathrm{D}$ input vector $\left[w_{x}, w_{y}, w_{v}\right]$, describing the wind-field components along the $x, y$ and vertical directions. 


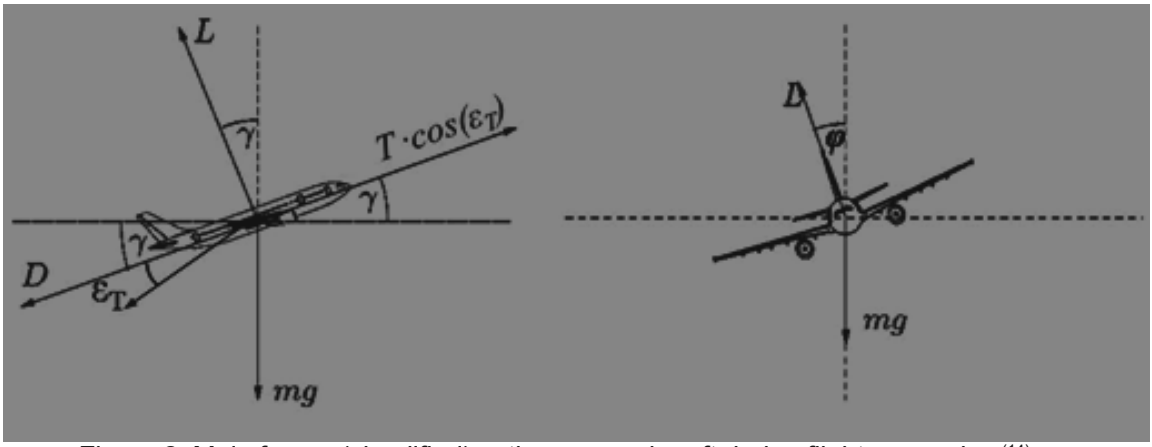

Figure 2. Main forces (simplified) acting on an aircraft during flight - overview ${ }^{(14)}$.

$$
\begin{gathered}
\dot{x}(t)=V_{T A S}(t) \operatorname{Cos} \Psi(t) \operatorname{Cos} \gamma(t)+w_{x}(t) \\
\dot{y}(t)=V_{T A S}(t) \operatorname{Sin} \Psi(t) \operatorname{Cos} \gamma(t)+w_{y}(t) \\
\dot{h}(t)=V_{T A S}(t) \operatorname{Sin} \gamma(t)+w_{v}(t) \\
\dot{V}_{T A S}(t)=\frac{T(t)}{m(t)} \operatorname{Cos} \varepsilon_{T}-\left(\frac{D(t)}{m(t)}+g \operatorname{Sin} \gamma(t)\right) \\
=\frac{T(t)}{m(t)} \operatorname{Cos} \varepsilon_{T}-\left(\frac{C_{D} S \rho(t)}{2} \frac{V_{T A S}^{2}(t)}{m(t)}+g \operatorname{Sin} \gamma(t)\right) \\
\dot{\Psi}(t)=\frac{L(t)}{m(t) V_{T A S}(t)} \operatorname{Sin} \varphi(t)+\tau T(t)+W_{x y}(t) \\
\frac{C_{L} S \rho(t)}{2} \frac{V_{T A S}(t)}{m(t)} \operatorname{Sin} \varphi(t)+\frac{\operatorname{Sin}\left(\gamma(t)+\varepsilon_{T}\right) \operatorname{Sin} \varphi(t)}{m(t) V_{T A S}(t) \operatorname{Cos} \gamma(t)} T(t)+W_{x y}(t) \\
\dot{m}(t)=-\eta(t) T(t)
\end{gathered}
$$

In Equation (1), $V_{T A S}$ is the air-speed, $D$ the aerodynamic drag, $C_{D}$ the drag coefficient, $S$ the total wing surface area obtained from BADA, $\rho$ the air density computed using the ISA model, $g$ the gravitational constant set to $9.80665 \mathrm{~m} / \mathrm{s}^{2}, L$ the lift, $C_{L}$ the lift coefficient and $\eta$ a factor describing the fuel consumption rate as a function of thrust, obtained from BADA Version 3.7, but modified as described in Section 4.5. Furthermore, $\varepsilon_{T}$ is the thrust-offset angle with respect to the wing-chord (as shown in Fig. 2), $W_{x y}$ the impact of the wind-force acting perpendicular to the flight-direction upon the rate of heading change, computed in Ref. 14 as:

$$
W_{x y}(t) \approx \frac{\rho(t)\left(S \operatorname{Sin} \varphi(t)+S_{b o d y / l a t}\right)}{V_{T A S}(t) m(t) \operatorname{Cos} \gamma(t)}\left(w_{x}^{2}(t)+w_{y}^{2}(t)\right) \alpha \operatorname{Sin}\left(\psi(t)-\operatorname{Arctan}\left(\frac{w_{y}(t)}{w_{x}(t)}\right)\right)
$$

where, $S_{b o d y / l a t}$ is the lateral surface area of the aircraft body, and

$$
\alpha=\left\{\begin{array}{l}
-1 \\
+1
\end{array}\right.
$$

depending on whether the aircraft turns into the wind or away from it respectively. The lateral aircraft body surface-area is not included in BADA, and is therefore estimated in the current 


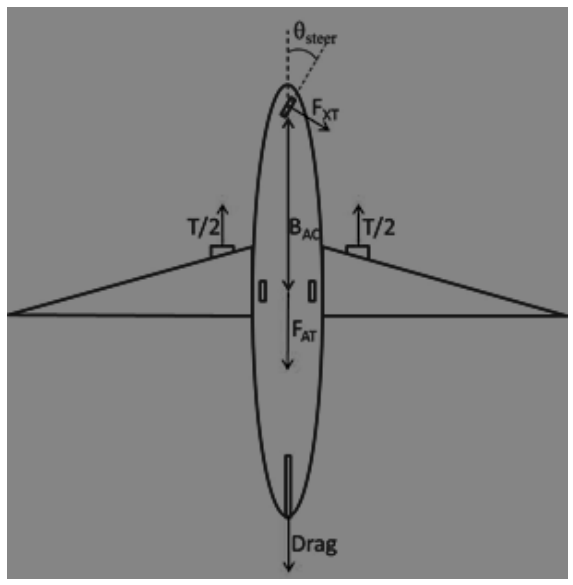

Figure 3. Main horizontal forces (simplified) acting on an aircraft during ground operations.

implementation of the model using the following formulation:

$$
S_{b o d y / l a t}=\Delta \cdot \Lambda
$$

where $\Delta$ is the aircraft diameter and $\Lambda$ is the aircraft length.

\subsection{Taxiing phase}

For the taxiing phase, the wind-factor was removed from the equations of motion. Friction was introduced and the change of heading modelled on the basis of the steering angle $\theta_{\text {steer }}$, rather than the aircraft bank-angle (see Fig. 3). The lateral motion of the aircraft on the ground is assumed to be exclusively controlled through the steering angle of the aircraft front gear. It is further assumed that sideways-slip of the aircraft front-gear is negligible, i.e. $F_{X T}$ is larger than the sum of the forces acting against it, a reasonable assumption under nominal taxiing (including environmental) conditions. Combining the state-parameters with the aircraft dynamics control and perturbation parameters, the aircraft motion is described on the basis of basic Newtonian laws as:

$$
\begin{gathered}
\dot{x}(t)=V_{G S}(t) \operatorname{Cos} \Psi(t) \operatorname{Cos} \gamma(t) \\
\dot{y}(t)=V_{G S}(t) \operatorname{Sin} \Psi(t) \operatorname{Cos} \gamma(t) \\
\dot{h}(t)=V_{G S}(t) \operatorname{Sin} \gamma(t) \\
\dot{V}_{G S}(t)=\frac{T(t)}{m(t)}-\left(\frac{C_{D} S \rho(t)}{2} \frac{V_{T A S}^{2}(t)}{m(t)}+g \operatorname{Sin} \gamma(t)+g F_{A T}(t)\right) \\
\dot{\Psi}(t)=\frac{V_{G S}(t)}{B_{A C}(t)} \operatorname{Sin} \theta_{\text {steer }}(t) \\
\dot{m}(t)=-\eta(t) T(t)
\end{gathered}
$$

In addition to the parameters previously defined, $V_{G S}$ is the ground-speed and $B_{A C}$ the aircraft baseline (as indicated in Fig. 3). 


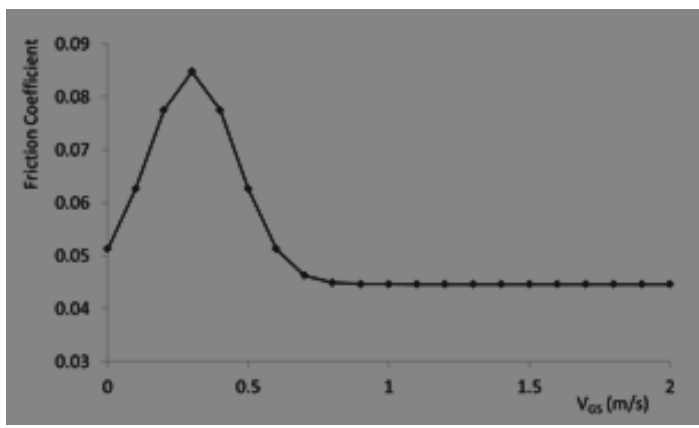

Figure 4. Friction coefficient as a function of ground speed for a B737-500.

The aerodynamic drag and effective friction between the tyres and the surface $F_{A T}$ act against the aircraft forward motion induced by the thrust. Furthermore, gravity also affects forward motion on inclined surfaces, accounted for by the third term for the acceleration in Equation (5). It should be noted that the thrust allocation to each engine is only illustrative in Fig. 3 (for the sake of clarity of the figure) and that the model uses the combined thrust at the centre of gravity of the airplane. Friction between tyres and the airport surface has been modelled for example in Ref. 18, where the following functional form is provided:

$$
F(t)=\alpha+\beta \cdot e^{\left(\frac{V(t)-\gamma}{\eta}\right)}
$$

where $\alpha, \beta, \gamma$ and $\delta$ are constants and $V$ is the speed. This expression was empirically modified in this paper to match the thrust requirements extracted from the flight data record (FDR):

$$
F_{A T}\left(V_{G S}(t)\right)=\frac{F_{A T}^{R}}{\left|\operatorname{Cos} \theta_{\text {steer }}(t)\right|}+A \cdot e^{B\left(\frac{V_{G S}(t)-V_{R}}{V_{R}}\right)^{2}}
$$

where $F_{A T}^{R}$ is the along-track friction coefficient if the aircraft was moving straight at infinite speed, $V_{G S}$ is the aircraft ground speed, $V_{R}$ the reference speed, and $A$ and $B$ are constants that are empirically determined. The model accounts for the fact that friction increases as the steering angle increases, reaching infinity when the steering angle reaches 90 degrees (i.e. perpendicular to the aircraft longitudinal axis). Furthermore, friction was found to initially increase when the aircraft transitions from a stationary state to a state of motion, and then decrease beyond a certain reference speed $V_{R}$. Figure 4 shows the friction coefficient as a function of aircraft ground speed empirically determined for the Boeing 737-500 aircraft analysed in this paper. On the basis of the data analysed (Section 5), the reference speed was found to be approximately $0 \cdot 3 \mathrm{~ms}^{-1}$.

The next section discusses the input data required for the aircraft dynamics system and the flight management system (discussed in Section 4).

\subsection{INPUT DATA - FLIGHT OBJECT}

A key requirement for successful trajectory based operations (TBOs) and trajectory management is the synchronisation of all relevant information between all relevant stakeholders. This is achieved by means of a harmonised set of information, encapsulated in the flight object ${ }^{(3)}-\mathrm{FO}$, proposed by Ref. 15. The FO consists of the following elements: the initial aircraft state, the operational 
intent and the aircraft intent, all part of the flight script, as summarised in Fig. 5. Additionally, the FO is expected to contain data relating to the flight identification, the original flight plan and the aircraft-computed 4D trajectory.

\subsection{Operational intent}

On the basis of the definition of flight intent in Ref. 3, this paper describes operational intent (OI) as the initial conditions and the set of constraints and user preferences that must be adhered to in the generation of the predicted trajectory. The terminology 'operational intent' is used rather than 'flight intent' to capture the airport surface movement phase of operation. In Ref. 14, operational intent is represented by a sequence of trajectory change points (TCPs) which implicitly describe the trajectory constraints, including aircraft, airport and airspace constraints and operator preferences. These TCPs are defined by a:

- horizontal and vertical position in terms of latitude, longitude and height;

- required time of overfly (RTO), describing the exact time at which the aircraft is required to fly-over or fly-by the TCP;

- required initial ground speed (RIGS), describing the speed at which the aircraft is required to fly at the TCP;

- turn-mode (TM), describing whether the aircraft is expected to 'fly-over' or 'fly-by' the TCP;

- holding mode (HM), describing whether the TCP belongs to a 'holding area';

- expedited descent mode (EDM), describing the spoiler configuration from the current until the next TCP.

\subsection{Aircraft intent}

Aircraft intent (AI) is defined as the aircraft operations plan that defines the set of actions that will enable the aircraft to meet the constraints and preferences defined in the $\mathrm{OI}^{(3)}$. This definition is in line with that in Ref. 14, where AI was defined as a structured set of instructions describing unambiguously the intended operational state of the aircraft over a given time-horizon in order to meet its flight intent. This paper adopts this definition, however modifies 'flight intent' to 'operational intent'. The set of instructions determines the evolution of the modelled dynamic state of the aircraft, computed by the core TP engine. The basic assumption is made that the aircraft follows each instruction to within the limits of its operational capability, taking into consideration surrounding environmental factors, such as wind, temperature and pressure. Each instruction is identified by a start-time and an end-time, specifying the length of time during which the given instruction is to be executed. A set of instructions forms an operation, which unambiguously determines the modelled aircraft motion during a given time interval.

A key premise of TP is that the variation in any of the state or control variables of the aircraft is continuous and smooth, as would be expected during actual operations. For example, when an aircraft follows a complex path, it is important that continuity is assured, not only in the position domain but also in the control domain. For lateral control, this means that for example the bankangle and the heading angle variations cannot present any step-wise discontinuity.

It is assumed in this paper that an aircraft follows the trajectory during airborne phases of operation by adjusting exclusively its bank-angle for lateral control; a combination of thrust and flight-path angle for speed control and a combination of flight-path angle and thrust for vertical control. During the ground phases, it is assumed that an aircraft uses exclusively the steering angle 


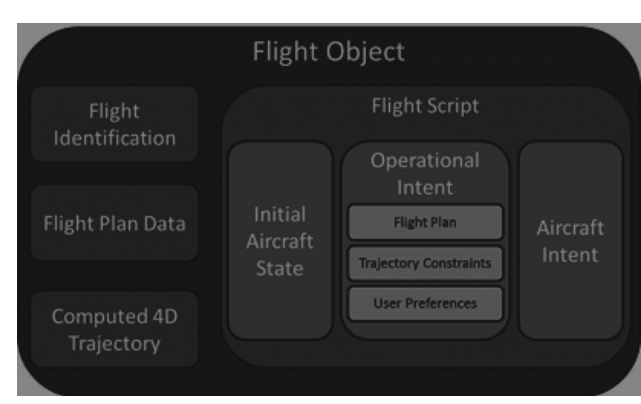

Figure 5. Flight object, adapted from Ref. 15.

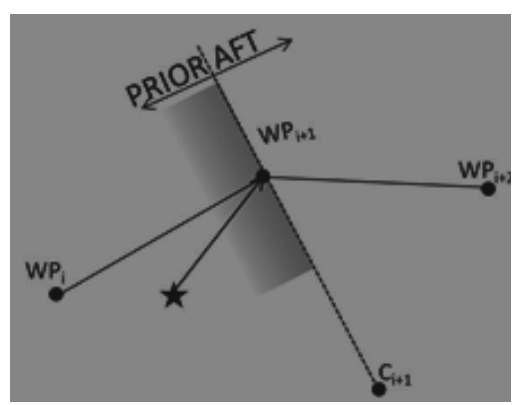

Figure 6. TCP update condition - complex turns.

of the front wheel for lateral control and thrust for speed control.

In order to determine the instantaneous aircraft control configuration (i.e. thrust, bank angle, flight path angle and drag) required, four parameters need to be computed by the FMS: the flight segment delimited by two TCPs (discussed in Section 3.2.1), the required speed and rate-of-climbdescent (discussed in Section 3.2.2), and the required turn-geometry for complex manoeuvres (discussed in Section 3.2.3). This information will be used by the FMS to determine the thrust, lateral control, vertical control and drag configuration requirements in Section 4.

\subsubsection{TCP update}

This paper determines the transition between TCPs on the basis of established operational procedures. In the author's prior work, a distinction was made between fly-over and fly-by modes, setting requirements on the relative angle and the anticipation-distance to the next TCP respectively. This paper complements these conditions and introduces the update condition for complex turns as follows: the aircraft is expected to fly over each of the TCPs which are part of the complex turn (similar to the fly-over condition), i.e. to within operational errors, the aircraft is expected to pass through $W P_{i}, W P_{i+1}$ and $W P_{i+2}$ in Fig. 6. The aircraft initially targets $W P_{i+1}$ until it has crossed the vertical plane perpendicular to the current segment and passing through the current turn-centre $\left(C_{i+1}\right)$ and $W P_{i+1}$. This approach ensures that the aircraft seamlessly passes between segments in the presence of potential offsets with respect to its nominal trajectory.

\subsubsection{Speed and rate-of-climb-descent requirements}

The speed and rate-of-climb-descent (ROCD) requirements developed in this paper are based on the model in Ref. 14. For the taxiing phase, the wind-factor is eliminated from the computation of the speed requirements because the relevant parameter is the ground speed and not the true airspeed. Moreover, it is assumed that during taxiing, friction is significantly larger than the impact of the wind upon the speed of the aircraft and that lateral friction of the tires is sufficient to counteract the lateral impact of the wind. The wind-factor is taken into consideration during the take-off roll and subsequent phases until the taxiing phase at the destination airport.

\subsubsection{Complex manoeuvres}

For complex manoeuvres, such as turns with varying turn-radii and/or altitudes, past approaches have specified turns in terms of a turn centre latitude and longitude as well as a turn radius ${ }^{(16)}$. In order to reduce the flight-script information (and thereby reduce bandwidths requirements for the transmission of intent information between relevant stakeholders), this paper shows that it is 


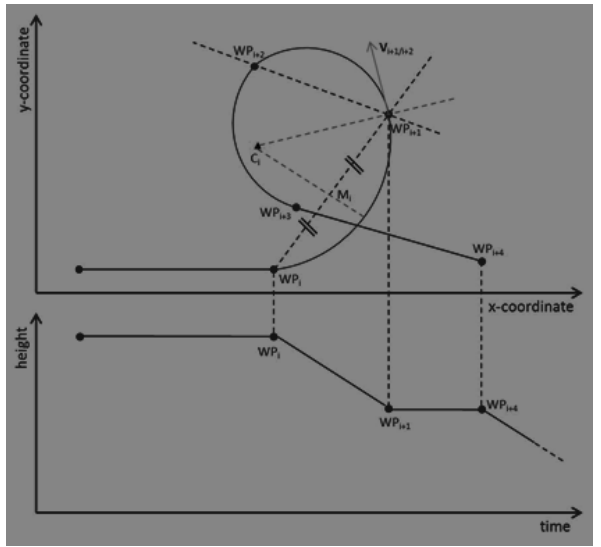

Figure 7. Complex descent manoeuvre: horizontal (top) and vertical (bottom) views.

possible to uniquely determine the aircraft intent during a complex procedure simply by specifying that a given waypoint marks the beginning of a constant radius turn, without the need to actually specify the turn-radius or turn-centre in the flight plan. This information is captured by the TM variable (see Section 3.1) which, in the version of the TP developed in this paper, describes the turn-status at a TCP as 'fly-over' or 'fly-by', and as 'nominal' or 'complex'.

Figure 7 shows a typical complex manoeuvre, in the horizontal (top) and the vertical (bottom) planes. The aircraft reaches $W P_{i}$ on a straight-line segment, it then enters a constant radius descending turn until it reaches $W P_{i+1}$, upon which it enters a second constant radius turn levelflight with a radius different from the previous one. Upon reaching $W P_{i+2}$, the aircraft enters yet another constant radius turn until it reaches $W P_{i+3}$, at which point it resumes a straight-line level profile, initiating a new straight-line descent at $W P_{i+4}$. This is a typical scenario in the TMA, and includes the possibility of holding manoeuvres. The turn-centre $C_{i}$ and turn-radius for the complex turn can be computed from the $3 \mathrm{D}$ co-ordinates $\left(x_{i}, y_{i}, z_{i}\right)$ of sequential waypoints along the intended flight path. In order to ensure that there are no discontinuities (e.g. in the bank-angle requirements) between subsequent segments, the turn-centre $3 \mathrm{D}$ position is uniquely determined by the intersection of three planes. Four cases must be distinguished if the current waypoint ( $W P i)$ is part of a constant-radius turn (CRT) (see Table 1):

The turn centre is computed from the intersection of one horizontal and two vertical planes:

- horizontal plane at the mid-altitude between $W P_{i}$ and $W P_{i+1}$ (applicable to all cases);

- vertical plane perpendicular to the:

o vector linking $W P_{i}$ and $W P_{i+1}$ containing the mid-point $M_{i}$ of the segment (Case 1);

o median angle subtending the segment linking $W P_{i}$ and $W P_{i+1}$ and the segment linking $W P_{i+1}$ and $W P_{i+2}$. (Cases 2, 3 and 4);

- Vertical plane perpendicular to the:

o tangent at $W P_{i}$ and passing through $W P i$ (Cases 1 and 2);

o vector linking $W P_{i}$ and $W P_{i+1}$ containing the mid-point $M_{i}$ of the segment (case 3);

o median angle subtending the segment linking $W P_{i+1}$ and $W P_{i}$ and the segment linking $W P_{i}$ and $W P_{i+1}$ (Case 4). 


\section{Table 1 \\ Complex procedure turn scenarios; $T C P_{i-1}=$ previous TCP; $T C P_{+1}=$ next TCP; RT = regular turn

$\begin{array}{lll} & \boldsymbol{W P}_{i-1} & \boldsymbol{W P}_{i+1} \\ \text { Case 1 } & \text { RT } & \text { RT } \\ \text { Case 2 } & \text { RT } & \text { CRT } \\ \text { Case 3 } & \text { CRT } & \text { RT } \\ \text { Case 4 } & \text { CRT } & \text { CRT }\end{array}$

The turn-radius is then computed from the distance between the turn-centre and the location of the next waypoint $W P_{i+1}$ :

$$
R=\sqrt{\left(x_{i+1}^{2}-C_{x}^{2}\right)+\left(y_{i+1}^{2}-C_{y}^{2}\right)+\left(z_{i+1}^{2}-C_{z}^{2}\right)}
$$

where $\left(x_{i+1}, y_{i+1}, z_{i+1}\right)$ are the co-ordinates of $W P_{i+1}$ and $\left(C_{x}, C_{y}, C_{z}\right)$ those of the turn-centre.

\subsection{FLIGHT MANAGEMENT SYSTEM}

The FMS uses information from the aircraft intent determined in Section 3.2 to compute four control variables as described in the following subsections: thrust (Section 4.1), bank-angle (Section 4.2), flight-path-angle (Section 4.3) and drag (Section 4.4). Each is adjusted considering the aircraft instantaneous state and aircraft-intent, with the goal to minimise deviations from the intended 4D trajectory (i.e. the operational intent). The control variable requirements are checked against aircraft operational limits which are derived from the BADA 3.7 model. While the model in this paper uses operational limits based on the BADA 3.7 model, it does not (with the exception of the drag) use the BADA nominal settings. Instead, the model computes the 'nominal settings' on the basis of the instantaneous predicted aircraft state (from the previous iteration of the TP loop) and the aircraft intent information and ensures that the target settings are within the operational limits of the aircraft. Additionally, the FMS determines the aircraft mass, on the basis of the fuel-consumption rate, discussed in Section 4.5.

\subsection{Thrust}

\subsubsection{Airborne phases}

Using basic Newtonian laws of motion, the required thrust is computed in Ref. 14 on the basis of the sum of forces resulting from nominal drag (computed using the BADA Version 3.7 inferred drag parameters), the required acceleration a and the gravitational force $g$ acting on the aircraft during the climb and descent:

$$
T(t)=T_{\text {cruise }}(t)+m(t) \quad g \operatorname{Sin} \gamma(t)+m(t) \quad a(t)
$$

where $\gamma$ is the required flight-path-angle and $T_{\text {cruise }}$ the thrust under level-flight and constant speed conditions, essentially determined by the drag acting on the aircraft:

$$
T_{\text {cruise }}(t)=\frac{C_{D} \rho(t) S}{2} V_{T A S}^{2}(t)
$$


A comparison of the maximum thrust capability specified in the BADA Version 3.7 model $^{(17)}$ and that from the FDR analysed in this paper (see Section 5) revealed a significant discrepancy $(\sim 12 \%)$. In this paper, the aircraft performance limit was therefore corrected to reflect actual aircraft performance capabilities as inferred from the FDR.

\subsubsection{Ground phases}

For the ground-phase of operations, an additional consideration for the thrust requirements is the friction between the aircraft tyres and the ground. In this paper, the additional thrust requirement due to friction is classically modelled as:

$$
T_{\text {friction }}(t)=m(t) \cdot g \cdot F_{A T}(t)
$$

where $F_{A T}$ is given by Equation (7). Using basic Newtonian physics, the total thrust requirement is then computed as:

$$
T(t)=\frac{C_{D} S \rho(t) V_{T A S}^{2}(t)}{2}+m(t) \cdot\left(g \operatorname{Sin} \gamma(t)+a(t)+g F_{A T}(t)\right)
$$

Figure 8 compares the actual thrust from the FDR (see Section 5) to the predicted thrust as a function of time. As can be seen, there is a significant divergence between the two curves at around $t=125 \mathrm{~s}$ and $t=370 \mathrm{~s}$. The former can be attributed to the lack of modelling of the start-up sequence of the engine. The latter is due to the fact that the aircraft is predicted to arrive earlier at the runway threshold than it does in reality and therefore starts its take-off sequence (i.e. increases its thrust) early as well. Given the larger time available to reach the next waypoint, less thrust is initially required. The detailed reasons for the early arrival are still under investigation, however potential contributing factors include imperfections in the friction model and the assumption that surface gradients are uniform between waypoints. Future work is expected to address these issues.

\subsection{Bank angle}

\subsubsection{Airborne phases}

Lateral control for complex manoeuvres is significantly different from phases of operation where the aircraft is simply attempting to track a straight-line path or relatively small variations in heading. Complex procedures require the aircraft to follow the appropriate arc. These are complicated by the rapidly varying impact of the wind-field upon the aircraft turn-radius, which is significantly dependent upon the relative wind-direction. This paper develops a novel approach for lateral guidance during constant radius-turns and complex procedures. A key innovation lies in the computation of the heading error (HE) and cross-track error (CTE) for complex procedures, which are no longer measured with respect to a straight line linking two waypoints but with respect to a curved path.

\section{Heading and cross-track errors}

The required aircraft heading during a complex turn is computed as the instantaneous tangent to the arc of circle subtending the current and next waypoints (see Fig. 9). The tangent vector $\bar{T}$ is computed as the line in the horizontal plane perpendicular to the vectors joining the turn-centre $\bar{C}$ and the current aircraft position $\bar{X}_{A C}$ : 


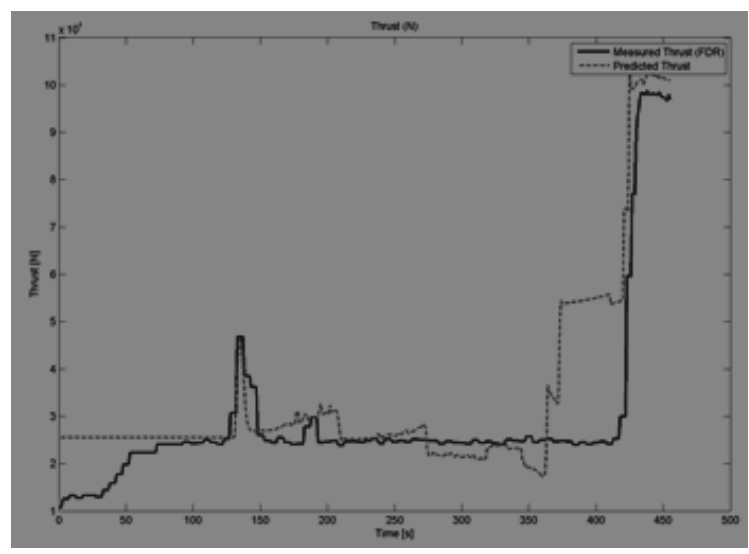

Figure 8. Thrust: recorded (solid line) and predicted (dashed line).

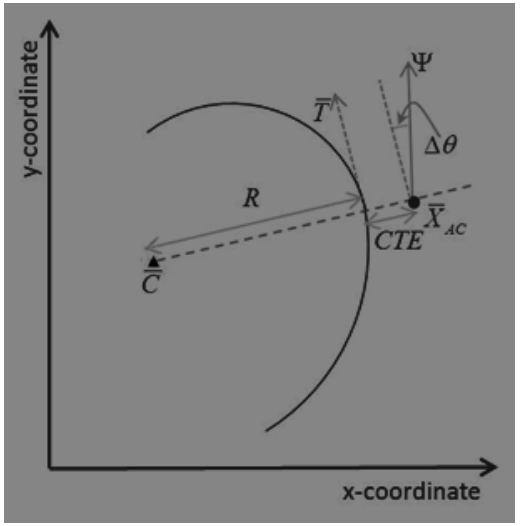

Figure 9. Lateral errors during complex manoeuvres.

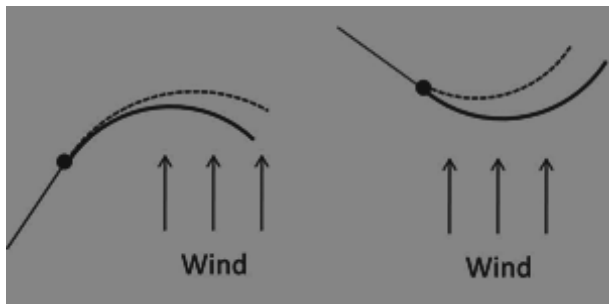

Figure 10. Impact of wind on turn-radius (continuous line: desired trajectory; dotted line: actual trajectory in the absence of wind-corrections).

$$
\bar{T}(t)=\bar{X}_{A C}(t)-\bar{C}(t)-90
$$

It is corrected for the wind-field by subtracting the wind-field vector from the tangent vector. The $\mathrm{HE}$ is then computed as:

$$
\Delta \theta(t)=\bar{T}(t)-\Psi(t)
$$

where $\Psi$ is the instantaneous aircraft heading. The aircraft cross-track error CTE is computed as the difference in distance between the aircraft and the turn-centre, and the instantaneously required turn-radius, i.e.:

$$
\operatorname{CTE}(t)=\left|\bar{X}_{A C}(t)\right|-R(t)
$$

\section{Required bank-angle}

The required bank-angle is a function of the CTE and $\mathrm{HE}$ as well as the required turn-radius and surrounding wind-field: turning upwind requires a significant steeper bank-angle, while turning downwind a significantly shallower bank-angle (see Fig. 10). The linear controller for the bank angle $\varphi$ is mathematically expressed as:

$$
\varphi(t)=k \cdot\left[\Delta \theta(t)-D(t) \cdot \beta(t)+f\left(R(t), W_{R}(t)\right)\right]
$$

where $k$ is a constant, $D$ is the turn-direction relative to the wind, $W_{R}$ is the wind-related factor and 


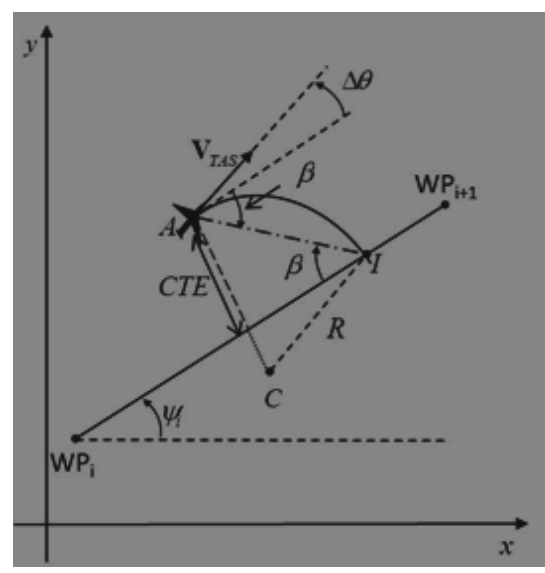

Figure 11. Lateral guidance - proposed model: adapted from Ref. 14.

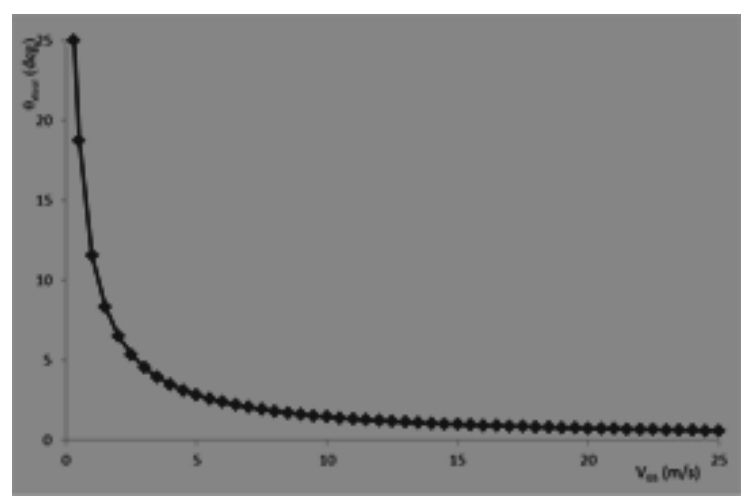

Figure 12. Nominal steering angle as a function of aircraft ground speed.

$\beta$ is defined as the difference between the nominal heading of the current segment and the vector pointing from the current aircraft location to point 'I', where 'I' is defined as the intersection of the current segment with the arc of circle of a nominal turn initiated by the aircraft from its current location A at its maximum angle of bank (see Fig. 11). The bank-angle is estimated as:

$$
\varphi(t)=k \cdot\left[\Delta \theta(t)-D(t) \cdot \beta(t)+\operatorname{Arctan}\left(\frac{D(t)}{g} \cdot\left[\frac{V_{T A S}^{2}(t)}{R(t)}+\frac{W_{R}(t)}{m(t)}\right]\right)\right]
$$

where $g$ is the gravity constant, $V_{T A S}$ the true airspeed and $m$ the aircraft mass. The wind-factor $W_{R}$ is estimated as:

$$
W_{R}(t) \approx W_{H Z}^{2}(t) \operatorname{Sin}\left(\bar{\Psi}(t)-\bar{W}_{H Z}(t)\right) \cdot \rho(t) \cdot\left(S \cdot|\operatorname{Sin} \varphi(t)|+S_{\text {lat }}\right) \cdot D(t)
$$

where $W_{H Z}$ is the horizontal wind-vector, $\rho$ the air density, $S$ the wing surface area and $S_{\text {lat }}$ the lateral surface area.

\subsubsection{Ground phases}

Lateral control is divided between straight-line and curved segments. For the former, the role of lateral control is to provide small adjustments to keep the aircraft on the taxiway centreline. During the airborne phases of operation, a nominal bank-angle provided within BADA Version 3.7 was assumed, irrespective of aircraft configuration or speed. However, this is not valid on the ground, where the nominal steering angle is a function of the aircraft taxiing speed, becoming progressively smaller at higher speeds. This paper empirically computes the nominal steering angle as a function of ground-speed as:

$$
\theta_{\text {steer }}^{\text {nom }}(t)=\theta_{\text {steer }}^{R} \frac{2 \cdot V_{R}}{V_{G S}(t)+V_{R}}
$$

where $\theta_{\text {steer }}^{R}$ is the steering angle at the reference speed $V_{R}$. Figure 12 shows that the nominal steering angle becomes rapidly smaller with increasing ground-speed, as one would expect from an operational perspective. 
For the case of curved segments, the target radius of turn $R_{F P}$ is computed as for the airborne phases of operation from the operational intent information. The desired steering angle uses a linear controller to determine the steering angle requirements to minimise the HE and CTE on the ground. The approach is similar to the one used for the airborne phases of operation. The assumption is made that lateral friction on the aircraft tyres is significantly larger than the side-wide force exerted by the wind, a key requirement for safe aircraft operations. Under nominal tracking conditions, the steering angle is then estimated as:

$$
\theta_{\text {steer }}(t)=k \cdot(\Delta \theta(t)+\beta(t))
$$

where, as for the airborne phase, $\beta$ is a pseudo-heading error resulting from the cross-track error. Using a similar approach to that in Ref. 14, on the basis of basic geometrical considerations, lateral guidance involving constant radius turns is modelled in this paper as a linear controller similar to the constant radius turns of the airborne phase of flight:

$$
\theta_{\text {steer }}(t)=k \cdot\left[\Delta \theta(t)-D(t) \cdot \beta(t)+D(t) \cdot \operatorname{Arctan}\left(\frac{B_{A C}}{R_{F P}(t)}\right)\right]
$$

\subsection{Flight path angle}

\subsubsection{Airborne phase}

The model proposed in Ref. 14 computes the FPA based on the ROCD and ground speed $V_{G S}$ requirements, taking into consideration the required allocation of thrust to the rate-of-change of height and to the change in speed to adhere to the intended flight-path. These requirements are determined from the aircraft instantaneous state and aircraft intent requirements. The FPA $\gamma$ is then geometrically determined as:

$$
\gamma(t)=\operatorname{Sin}^{-1}\left(\frac{R O C D(t)}{V_{G S}(t)}\right)
$$

This paper modifies this model to take into consideration the impact of the vertical wind component $W_{v}$ :

$$
\gamma(t)=\operatorname{Sin}^{-1}\left(\frac{R O C D(t)-W_{v}(t)}{V_{G S}(t)}\right)
$$

\subsubsection{Ground phase}

On the ground, the vertical movement of the aircraft is constrained by the hard-surface. Therefore, the vertical motion is defined by the gradient of the airport surface, which is computed from the three-dimensional waypoint co-ordinates:

$$
\dot{h}(t)=V_{G S}(t)\left(\frac{\Delta h_{W P}(t)}{d_{W P}(t)}\right)
$$

where $\Delta h_{W P}$ is the difference in height between two subsequent waypoints and $d_{W P}$ is the corresponding distance. 


\subsection{Drag}

Drag is a key driver of trajectory prediction performance. It affects amongst others the thrust required to meet a given speed profile. In turn, the thrust determines the fuel-flow rate, which determines the mass, which itself affects the thrust requirements.

\subsubsection{Airborne phase}

BADA Version $3.7^{(17)}$ expresses the coefficient of drag for phases of flight other than approach and landing, as:

$$
C_{D}=C_{D 0}^{\text {climb/cruise }}+C_{D 2}^{\text {climb/cruise }} C_{L}^{2}
$$

where $C_{D 0}^{\text {operational phase }}$ and $C_{D 2}^{\text {operational phase }}$ are constants defined in BADA and $C_{L}$ is the coefficient of lift. For approach and landing, the following expressions are used respectively:

$$
\begin{gathered}
C_{D}=C_{D 0}^{\text {approach }}+C_{D 2}^{\text {approach }} C_{L}^{2} \\
C_{D}=C_{D 0}^{L D G}+C_{D 2}^{L D G} C_{L}^{2}+C_{D}^{\Delta L D G}
\end{gathered}
$$

where $C_{D 0}^{\triangle L D G}$ is a constant that reflects the additional drag due to the landing gear. It was found in this paper that the BADA drag base coefficient $C_{D 0}^{\text {operational phase }}$ had to be increased by $19 \%$ for the climb and cruise phases of operation in order to simultaneously minimise the errors between the predicted aircraft state and control parameters and the corresponding parameters recorded by the FDR. The effective drag appears thus to be larger than predicted by BADA. Potential reasons include variability between same aircraft types, for example as a result of differences in the number and/or location of antennae, and/or differences in the aircraft skin, which can significantly affect friction $^{(19)}$.

\subsubsection{Ground phase}

There are no drag specifications in the BADA for ground operations. On the ground, the aircraft has a configuration similar to that of the climb phase of flight, with the exception of the additional drag from the landing gear. Therefore the drag coefficient for ground operations is modelled in this paper as follows:

$$
C_{D}=C_{D 0}^{\text {climb }}+C_{D 2}^{\text {climb }} C_{L}^{2}+C_{D 0}^{\Delta L D G}
$$

\subsection{Fuel-consumption rate}

\subsubsection{Airborne phase}

BADA provides fuel-consumption rate (FCR) parameters that enable the computation of the instantaneous aircraft mass once the initial aircraft mass is known. However, significant discrepancies were found between the data provided by the FDR and BADA Version 3.7. As a result, the approach was taken to adjust the FCR parameters with a correction parameter $C_{\text {phase }}$ for each of the phases of aircraft operation to reflect the FDR data:

$$
C_{D}=C_{D 0}^{\text {climb }}+C_{D 2}^{\text {climb }} C_{L}^{2}+C_{D 0}^{\Delta L D G}
$$




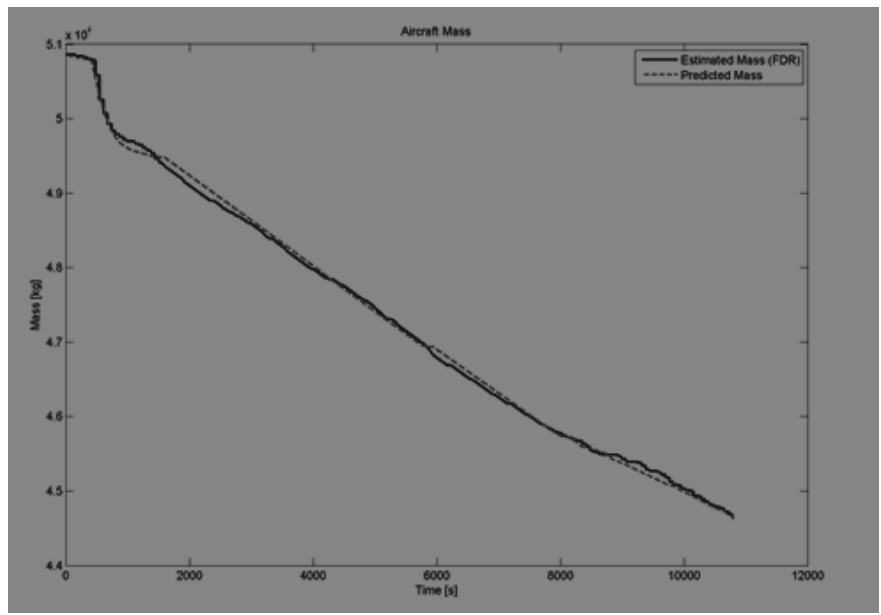

Figure 13. FDR (solid line) versus predicted (dashed line) mass.

where phase is the airborne operational phase of the aircraft, i.e. climb, cruise and descent. The following values were found for the B-735 flight analysed within the scope of this paper:

$$
\begin{aligned}
& C_{\text {climb }}=2 \cdot 35 \cdot e^{\frac{8000-h}{8000}} \\
& C_{\text {cruise }}=0.84 \\
& C_{\text {descent }}=2 \cdot 00
\end{aligned}
$$

A potential explanation for the significant difference between the BADA parameters and actual parameters recorded by the FDR is the aging of aircraft engines and/or the impact of environmental parameters (such as pressure) upon the FCR. Further research, based upon the analysis of extensive FDR data, is required to determine the validity of these hypotheses.

\subsubsection{Ground phase}

There are no available FCR data for airport surface movement. As a result, this paper took the approach to derive these FCR data on the basis of the mass recordings from the flight-data records:

$$
F C R_{A S M}=F C R_{\text {climb }}^{B A D A} \cdot 1 \cdot 8
$$

\subsubsection{Recorded versus predicted mass}

Figure 13 shows the mass evolution as a function of time, both from the flight-data records and the prediction from the TP engine using the FCR model developed in this paper. The mean difference is $6 \mathrm{~kg}$, the standard deviation $69 \mathrm{~kg}$ and the maximum absolute value of the difference $279 \mathrm{~kg}$, translating into an error of less than $0 \cdot 6 \%$. The difference can be attributed to errors in the fuel-consumption rate model, as well as natural imperfections associated with the TP model. For example, if the aircraft is predicted to arrive early at the take-off threshold, the take-off thrust is applied early as well, leading to a predicted fuel consumption rate that is different from the actual fuel-consumption rate as a function of time. However, the overall performance is extremely good. 

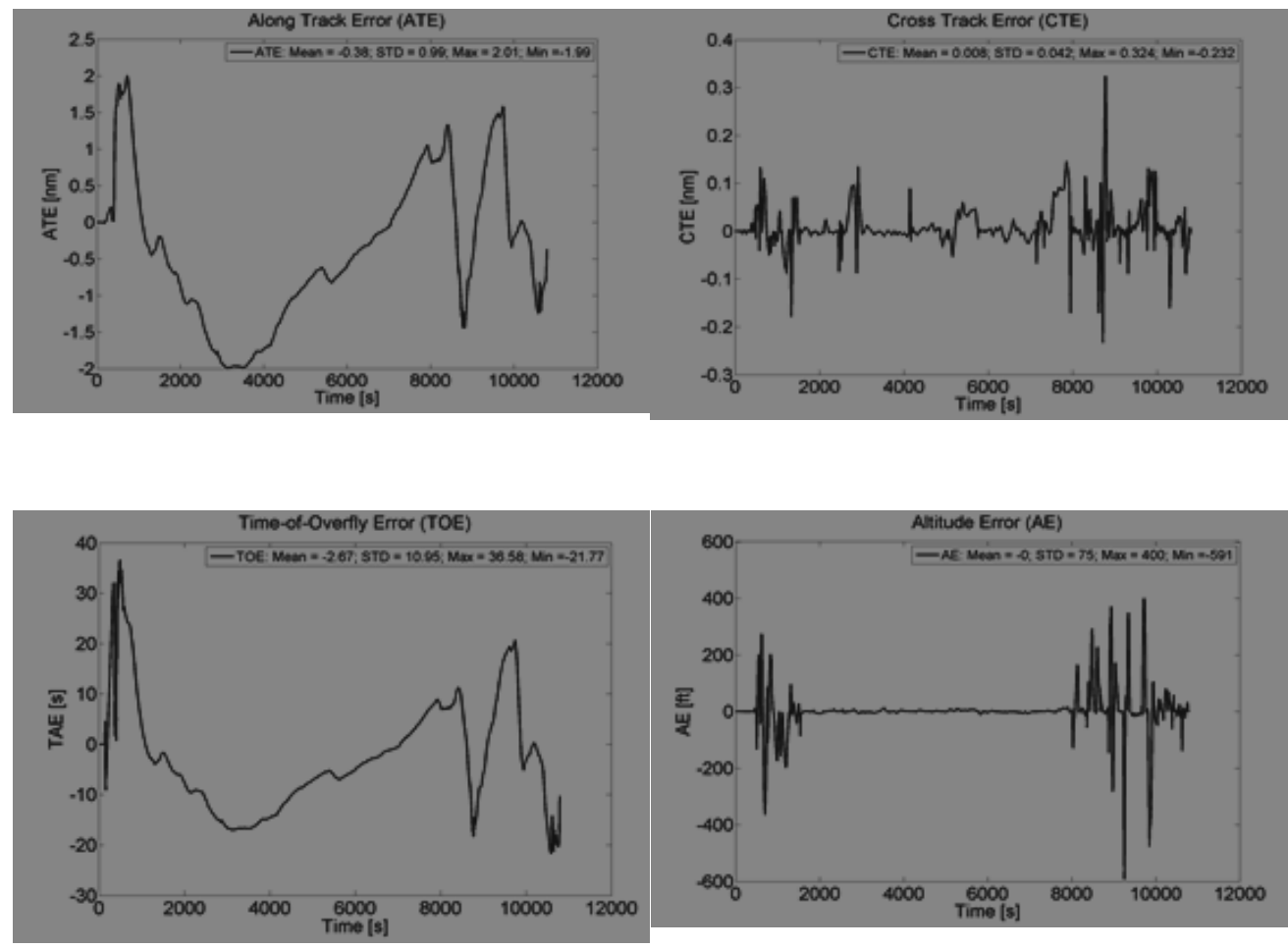

Figure 14. State parameter errors (ATE, CTE, TOE and AE) as a function of time.

\subsection{PERFORMANCE CHARACTERISATION}

The performance of the TP model has been evaluated using a single flight trial in European airspace. Detailed FDR were provided by EUROCONTROL ${ }^{(20)}$. These include aircraft 4D position, attitude, thrust settings, speed (true and calibrated air speeds, ground speeds and ROCD), mass and environmental conditions (wind-field, temperature and pressure) as a function of time, for a three-hour Boeing 737-500 aircraft flight. This is the same reference trajectory that was used to evaluate the performance of the model in Ref. 14. The wind strength and direction are extracted from onboard measurements, interpolated to each instantaneous predicted aircraft position, i.e. the predicted wind is the same as the measured wind to within interpolation errors. All the necessary aircraft performance parameters were taken from the BADA Version 3.7 set $^{(17)}$. The choice of the BADA 3.x version was based upon the availability at the time of the research in this paper. These versions are known to suffer from inaccuracies in the fuel consumption rate, a fact that was confirmed in this paper. The higher level of performance of the BADA 4 model $^{(21,22)}$ is expected to address some of these issues. Additionally, aircraft diameter and length were taken as $3.54 \mathrm{~m}$ and $31.0 \mathrm{~m}$ respectively, resulting in a total lateral aircraft-body surface area of $109.74 \mathrm{~m}^{2}$. The waypoints were determined through a backward analysis of the recorded trajectory (as described in detail in Ref. 14) to ensure that deviations from the flight plan resulting from ATC instructions did not contribute to the TP model errors. TP accuracy is evaluated in terms of 4D position accuracy and the accuracy of other key parameters, discussed in turn below. 


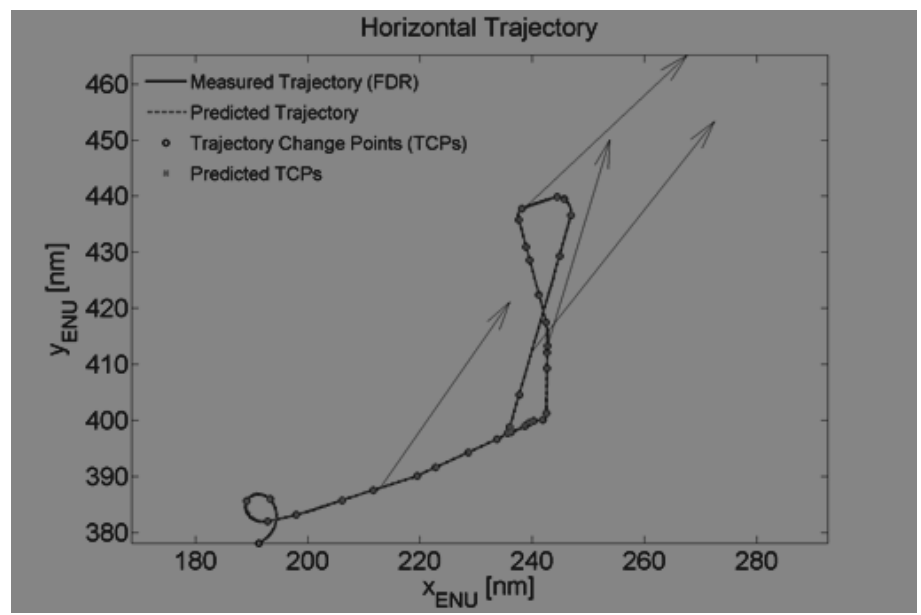

Figure 15. Horizontal trajectory - terminal manoeuvring area; arrows indicate the wind direction.

\subsection{D position accuracy}

The TP state-parameter accuracy is evaluated in terms of Euclidean Errors (EE), defined as the 3D distance between the actual aircraft position and the nominal position for each point in time. The $\mathrm{EE}$ are then projected along-track (determined from the recorded heading) and perpendicular to the recorded track in the horizontal and the vertical, to evaluate respectively the along track errors (ATE), CTE and altitude-errors (AE). Furthermore, the accuracy of the aircraft in meeting the RTOs is evaluated as time-of-overfly errors (TOE). These are computed as the difference between the nominal time and the actual time of the aircraft for each point along the recorded trajectory.

The accuracy of each parameter was analysed over the chosen operational segment (i.e. ASM, CLIMB, ENROUTE, DESCENT, TMA, or ALL) by computing the difference between the predicted trajectory and the actual trajectory flown by the aircraft (recorded by the FDR). Therefore, the TP errors incorporate also errors from the FDR. However, these are negligible given that they are recorded from onboard navigation sensors such as GPS, with accuracies that are at the metre-level. The difference can therefore be attributed to the TP errors. The following maximum absolute errors are observed under ideal conditions (i.e. no input uncertainties) for each of the phases of operation:

Figure 14 shows the time evolution of these state parameters. As shown in Table 2 and Fig. 14, the maximum CTE of the predicted trajectory never exceed $0.32 \mathrm{~nm}$ for any phase of operation. The maximum AE are $15 \mathrm{ft}$ for the enroute phase and around $300 \mathrm{ft}$ for the climb and descent, which exhibit similar behaviour. The maximum AE are larger for the TMA portion of the flight ( $\sim 60 \mathrm{ft})$ due to the rapid combined variation of heading and altitude, which causes a rapid variation in the wind-impact, especially during the procedure turn. For the climb and descent phases, the AE can be attributed to the fact that the vertical wind-fields are not known. The ATE error is largest for the TMA, with maximum values of $\sim 2 \cdot 2 \mathrm{~nm}$. During this phase of flight, the aircraft follows a complex path (shown in Figs 15 and 16) during which there are significant variations in the wind-direction, reflected in the significantly larger error distribution of the wind-field estimation. The arrows in Fig. 15 show the wind-direction for a select sample of points along the trajectory. Note that while the ATE is largest for the TMA, the TOE is largest for ASM due to the significantly lower aircraft speeds. This is due to large variations in aircraft speed during the take-off. Any small discrepancy in timing results in large variations in predicted aircraft speed at a given point in time. 


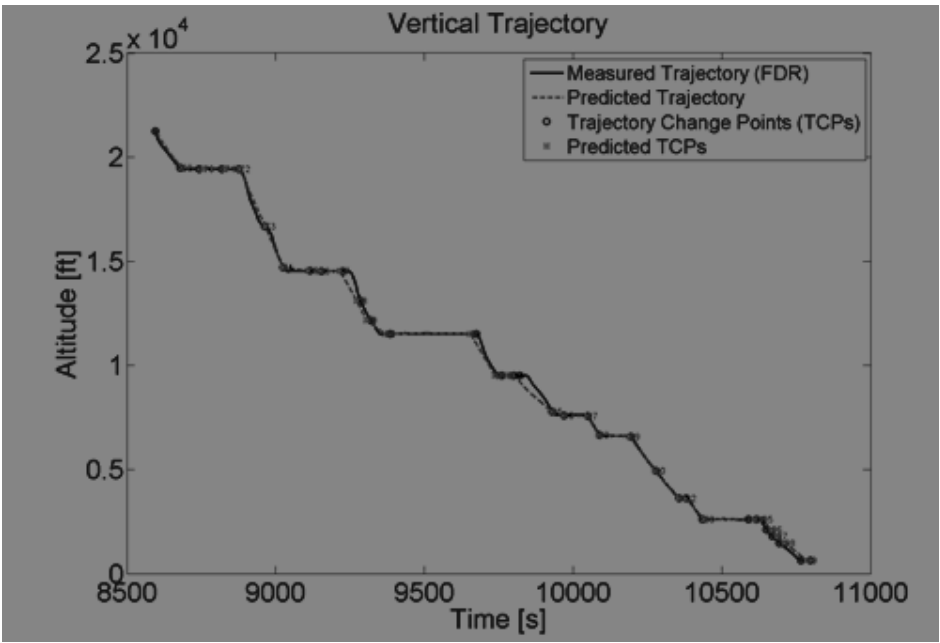

Figure 16. Vertical trajectory - terminal manoeuvring area.

Table 2 shows that there are differences between the maxima of the total trajectory and the maxima of the individual segments combined. For example, the maximum ATE of the TMA is approximately $0 \cdot 2 \mathrm{~nm}$ larger than the maximum ATE of the overall trajectory. Similarly, the maximum TOE of the total trajectory is about $3.4 \mathrm{~s}$ larger than the maxima of the individual segments. This is due to the fact that for the analysis of each segment, the initial configuration error was set to zero. In practice, for the analysis of the total trajectory, the initial configuration error of all phases other than the ASM is different from zero. However, as expected, the discrepancies are relatively small.

A detailed performance comparison with other TP tools was unfortunately not possible given the lack of gate-to-gate capabilities of TP tools in the public domain. However, prior work by the authors ${ }^{(14)}$ has shown that their TP tool performance for the enroute phase of operation was approximately an order of magnitude better than prior state-of-the-art.

To note is that the analysis in this paper has focused on TP performance under ideal conditions, i.e. when all input data are accurately known. It is thus a measure of the performance of the TP model rather than a measure of the TP tool performance (which includes both the TP model and input data performance). The TP tool performance is expected to degrade in the presence of errors in the input parameters. This may for example have safety and efficiency implications if the TP tool feeds conflict detection and resolution tools for separation assurance. The impact of input data errors on TP performance is the subject of further research.

\subsection{Accuracy of other state parameters}

An important finding in this paper is that 4D position accuracy performance is not sufficient to guarantee uniqueness in the remaining state or the control parameters. In particular, the thrust and aircraft mass recorded by the FDR and the thrust and mass predicted by the TP model on the basis of the BADA drag parameters initially showed significant discrepancies, despite the fact that the 4D trajectory could be accurately predicted. To make the TP more operationally realistic, prediction of the remaining parameters must also be accurate. The discrepancies were traced back to incorrect coefficients in the drag and fuel-flow of the BADA Version 3.7 model. This paper determined these coefficients by minimising simultaneously the discrepancy between the 
Table 2

Performance results - state parameters (ideal conditions); bold - worst performance; grey - best performance

$\begin{array}{lccccc}\text { PHASE } & \text { ATE }[\mathbf{n m}] & \text { TOE }[\mathbf{s}] & \text { CTE }[\mathbf{n m}] & \text { AE }[\mathbf{f t}] & \text { Duration [s] } \\ \text { ASM } & 1 \cdot 5 & 33 \cdot 2 & 0 \cdot 02 & 1 & 456 \\ \text { CLIMB } & 1 \cdot 4 & 15 \cdot 2 & 0 \cdot 18 & 339 & 1,097 \\ \text { ENROUTE } & 2 \cdot 0 & 16 \cdot 8 & 0 \cdot 21 & 15 & 6,462 \\ \text { DESCENT (high) } & 0 \cdot 8 & 6 \cdot 7 & 0 \cdot 10 & 297 & 580 \\ \text { TMA (complex) } & 2 \cdot 2 & 28 \cdot 5 & 0 \cdot 32 & 593 & 2,202 \\ \text { TOTAL } & 2 \cdot 0 & 36 \cdot 6 & 0 \cdot 32 & 591 & 10,797\end{array}$

Table 3

Performance results - state and control parameters other than 4D position (ideal conditions)

$\begin{array}{lcccc}\text { PHASE } & \text { Thrust [\%] } & \text { Mass [\%] } & \text { TAS [kts] } & \text { ROCD [ft/min] } \\ \text { ASM } & 90 \% & 0 \cdot 1 \% & 72 & \text { N/A } \\ \text { CLIMB } & 12 \% & 0 \cdot 2 \% & 28 & 740 \\ \text { ENROUTE } & 12 \% & 0 \cdot 2 \% & 8 & 188 \\ \text { DESCENT } & 40 \% & 0 \cdot 1 \% & 34 & 716 \\ \text { TMA (complex) } & 114 \% & 0.2 \% & 36 & 990 \\ \text { TOTAL } & 58 \% & 0 \cdot 3 \% & 28 & 608\end{array}$

4D trajectories and the aircraft thrust and mass parameters. The performance of the following parameters was evaluated: thrust, mass, speed, and ROCD, as shown in Table 3, where the values are expressed at a 2 -sigma (95\%) level.

The results in Table 3 show that, overall, the agreements between recorded and predicted data is very good for the climb, enroute and descent phases of operation, typically showing maximum deviations of less than $15 \%$. Average thrust deviations during the climb phase is $6 \%$, en route $1 \%$ and descent $2 \%$. However, for the ASM phase, the maximum thrust and TAS errors are more significant. Average thrust errors for ASM were 25\% and for the TMA 4\%. For ASM, the errors can be attributed to the fact that the aircraft arrives ahead of schedule at the runway threshold where it starts its take-off roll. This results in the application of take-off thrust, much larger than the thrust required for taxiing, ahead of schedule. It also explains the observed TAS errors. Future work will refine the surface friction model to further improve surface movement prediction performance, and implement a time-based logic to prevent the aircraft from starting its take-off sequence early. For the TMA, rather large deviations in terms of thrust and ROCD are observed. These can mainly be attributed to the variable wind impact during the complex procedures in the TMA, as shown in Fig. 15. 


\subsection{CONCLUSIONS}

Trajectory prediction capabilities are crucial for underpinning the SESAR, NextGen and other future concepts of aircraft operation. This paper has developed a gate-to-gate trajectory prediction model which was shown to be able to realistically predict the trajectory of a B735 aircraft for all phases of operation. The analysis showed that in order to minimise the errors of state and control parameters other than the 3D position as a function of time, it was necessary to correct several BADA parameters. The extent to which these parameters require adaptation between aircraft of a given type and as a function of aircraft type will require further validation with more data, which were unavailable to the authors. Assuming that computational requirements can be met, the TP model developed in this paper can be used onboard aircraft in addition to ground-based systems, thereby providing a method for the holistic strategic as well as tactical optimisation of ATM by all relevant stakeholders, including airlines and air navigation service providers. The TP model is based on actual operational data, accounting for aircraft dynamics, and operational procedures and limitations. Evaluation of performance in terms of 4D position errors and errors in other key aircraft state and control parameters shows that the model is operationally realistic and able to accurately represent the aircraft 4D position as well as other aircraft parameters. The results suggest that the currently proposed performance targets for the TOE over a given point (set at $30 \mathrm{~s}-95 \%$ - for the enroute phase of flight) are expected to be met ${ }^{(23)}$.

\section{ACKNOWLEDGEMENTS}

The authors would further like to thankfully acknowledge the SESAR Joint Undertaking and EUROCONTROL for sponsoring the TESA project under which this research was carried out. The authors would further like to thankfully acknowledge the EUROCONTROL TP team for providing the reference validation data used in the analysis of this paper.

This work was co-financed by EUROCONTROL acting on behalf of the SESAR Joint Undertaking (the SJU) and the EUROPEAN UNION as part of Work Package E in the SESAR Programme. Opinions expressed in this work reflect the author's views only and EUROCONTROL and/or the SJU shall not be considered liable for them or for any use that may be made of the information contained herein.

\section{REFERENCES}

1. SJU, The European ATM Master Plan, (second edition), 2012, https://www.atmmasterplan.eu/.

2. FAA, NextGen Implementation Plan, 2013, https://www.faa.gov/nextgen/implementation/media/ NextGen_Implementation_Plan_2013.pdf.

3. MusialeK, B. et al Literature survey of trajectory predictor technology, 2010, TC-TN11/1.

4. Petricel, B. and Costelloe, C. First ATC support tools implementation (FASTI) operational concept, Edition 1.1, 2007.

5. PAIELLI, R. Tactical conflict resolution using vertical maneuvers in en route airspace, AIAA J Aircr, 2008, 45, (6).

6. Erzberger, H. and Heere, K. Algorithm and operational concept for resolving short range conflicts, 2008, 26th International Congress of the Aeronautical Sciences, Anchorage, Alaska, USA.

7. KonYAK, M. et al Improving ground-based trajectory prediction through communication of aircraft intent, AIAA Guidance, Navigation and Control, 2009, Chicago, IL, USA.

8. Romanelli, J. et al Climb trajectory prediction software validation for decision support tools and simulation models, ITEA J, 2009, 30, pp 481-491.

9. Marceau, G. et al Online learning for ground trajectory prediction, 2012, SESAR Innovation Days, Braunschweig. 
10. Christien, R. and Pugh, R. Sharing data could improve trajectory prediction, 2012, P5.5.2, http://www. eurocontrol.int/articles/sharing-data-could-improve-trajectory-prediction.

11. Glover, W. and Lygeros, J. A multi-aircraft model for conflict detection and resolution algorithm validation, HYBRIDGE, 2004, WP1 (Deliverable D1.3).

12. Fairley, G. and McGovern, S. A Kinematic/kinetic hybrid airplane simulator model, 2008, ASME International Mechanical Engineering Congress and Exposition, Boston, MA, USA.

13. KARR, D. et al Autonomous operations planner: A flexible platform for research in flight-deck support for airborne self-separation, 2012, 12th AIAA Aviation Technology, Integration, and Operations (ATIO), Indianapolis, USA.

14. Schuster, W., Porretta, M. and Ochieng, W. High-accuracy four-dimensional trajectory prediction for civil aircraft, Aeronaut J, 2012, 116, (1175).

15. Eurocontrol and FAA, White Paper - Common TP structure and terminology in support of SESAR \& NextGen, Eurocontrol/FAA Action Plan 16 Common Trajectory Prediction Capability, Edition 1.0, 2010.

16. Mondoloni, S. and KIRK, D. Proposed trajectory prediction and exchange information items for flight information exchange model (FIXM), 2012, http://www.fixm.aero/sites/default/files/dashboardfiles/ dashboard/gfi/MITRE\%2012.pdf.

17. Eurocontrol, User Manual for the Base of Aircraft Data (BADA), Version 3.7, 2009.

18. EASA, RuFAB - Runway friction characteristics measurement and aircraft braking, Vol 3, 2008, (EASA.2008/4).

19. SAdraey, M., Drag Force and Drag Coefficient, 2009, VDM Verlag Dr Mueller.

20. Eurocontrol, Reference Validation Data Base, 2009.

21. Eurocontrol, BADA 4.0, 2014, http://www.eurocontrol.int/services/bada.

22. Poles, D., Nuic, A. and Mouillet, V. Advanced aircraft performance modeling for ATM: Analysis of BADA model capabilities, 2010, Digital Avionics Systems Conference (DASC), Salt-Lake City, UT, USA.

23. EUROCAE, MASPS required navigation performance for area navigation, 2012, ED75B (Addendum) www.eurocae.net. 